\title{
Skin Lesion on the Cheek of a Child after Dentist Appointment
}

\author{
Cinzia Casu ${ }^{1 *}$ and Riccardo Botta ${ }^{2}$ \\ ${ }^{1}$ Private Dental Practice, Italy \\ ${ }^{2}$ Departement of Dentistry, IRCSS San Raffaele Hospital, Italy
}

*Corresponding author : Cinzia Casu, DDS, Private Dental Practice, Cagliari, Italy.

Received Date: December 06, 2018

Published Date: December 13, 2018

\section{Short Communication}

An 8-year-old boy went in my private dental practice for a control of a deciduous tooth. The child is in good general health and the parents did not report any health problems. The deciduous element in the right jawbone was extremely compromised and with sharp edges that was creating small wounds in the patient's mouth. It was decided to carry out the avulsion of the element. A mepivacaine anesthesia with 1: 100,000 adrenalines, in vials of $1.8 \mathrm{cl}$ was administrated. The local anesthesia was injected on the alveolar mucosa near the deciduous element. The extraction was performed with a straight lever within a few minutes. The post-surgical wound was medicated with cotton rolls and after 15 minutes the patient was discharged. 12 hours later the parents of the child called back to see the child who had a lesion on the right cheek, the same where the avulsion was performed. The patient showed an area of about $4.5 \mathrm{~cm}$ of larger diameter, abraded and brown. The child reported a burning sensation. The alveolar mucosa in the site of avulsion was in good condition, there were no lymph node swelling, the post-extraction site was not painful. The patient was not an allergic subject. A more detailed anamnestic investigation showed that the child did not feel the right cheek after anesthesia and had rubbed the back of his hand several times on the cheek and without knowing it, had caused the skin lesion. Application of Aloe vera gel 2 times a day for 7 days was prescribed to reduce burning sensation and to improve the healing.
We decided to document this case because it is an example of how important it is to provide detailed information to the little patient and to the parents especially after having done for the first time an anesthesia. In this way you can avoid traumatic injuries due to loss of nerve sensitivity and some concern from the dentist and patient [Figure 1].

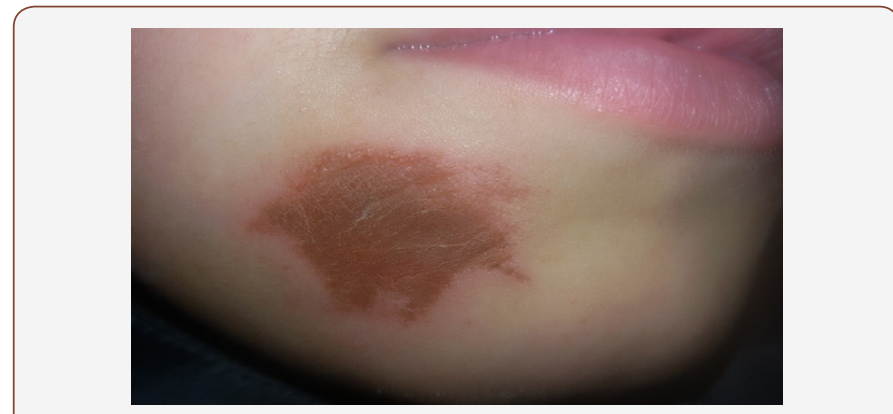

Figure 1: 8 years old male patient with cheek lesion after local anesthesia.

\section{Acknowledgement}

None.

\section{Conflict of Interest}

No conflict of interest. 